\title{
SYSTEM APPROXIMATION WITH GENERAL MEASUREMENT FUNCTIONALS
}

\author{
Holger Boche* \\ Technische Universität München \\ Lehrstuhl für Theoretische Informationstechnik
}

\author{
Ullrich J. Mönich ${ }^{\dagger}$ \\ Massachusetts Institute of Technology \\ Research Laboratory of Electronics
}

\begin{abstract}
The approximation of linear time-invariant (LTI) systems by sampling series is an important topic in signal processing. Recently, it was conjectured [1] and proved [2] that, for every sampling pattern that is a complete interpolating sequence, there exists a universal stable LTI system such that for every oversampling factor there exists a bandlimited input signal such that the approximation process, which is used to approximate the output signal of the LTI system, diverges. This instability of the approximation process shows a fundamental limit of sampling-based signals processing. However, as is shown in this paper, by using more general measurement functionals this divergence can be overcome.
\end{abstract}

Index Terms - bandlimited signal, linear time-invariant system, complete interpolating sequence, sampling, measurement functional

\section{INTRODUCTION}

Sampling theory plays a fundamental role in modern signal and information processing, because it is the basis for today's digital world [3]. The reconstruction of bandlimited signals from their samples is also essential for other applications and theoretical concepts $[4,5$, 6]. For an overview of existing sampling theorems see for example $[4,7,8]$.

Although the sampling theorems are very important on their own, they do not reflect the actual purpose of signal processing. The core task of signal processing is to process data. This means that, usually, the interest is not in a reconstruction of the sampled signal itself, but in some processed version of it. This might be the derivative, the Hilbert transform or the output of any other stable linear system $T$. In the general case the goal is to approximate the desired transform $T f$ of a signal $f$ by an approximation process, which uses only finitely many, not necessarily equidistant, samples of the signal $f$. Exactly as in the case of signal reconstruction, the convergence and approximation behavior is important for practical applications [9].

In this paper we consider bandlimited signals from the PaleyWiener space $\mathcal{P} \mathcal{W}_{\pi}^{1}$, which consists of bandlimited signals with absolutely integrable Fourier transform. In the case of oversampling

* This work was partly supported by the German Research Foundation (DFG) under grant BO 1734/22-1.

$\dagger$ U. Mönich was supported by the German Research Foundation (DFG) under grant MO 2572/1-1.

An extended version of this paper will be published as part of the book "New Perspectives on Approximation and Sampling Theory - Festschrift in honor of Paul Butzer's 85th birthday" in the Applied and Numerical Harmonic Analysis Series, Birkhäuser (Springer-Verlag). $(a>1)$, we can use the sampling series

$$
f(t)=\frac{1}{a} \sum_{k=-\infty}^{\infty} f\left(\frac{k}{a}\right) \phi\left(t-\frac{k}{a}\right),
$$

where $\phi$ are suitable reconstruction functions, to reconstruct $f$ from

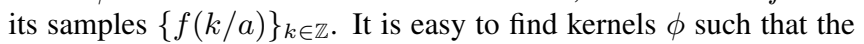
series in (1) is globally uniformly convergent.

However, in the system approximation problem an additional LTI system $T$ is present in the reconstruction process

$$
\frac{1}{a} \sum_{k=-\infty}^{\infty} f\left(\frac{k}{a}\right)(T \phi)\left(t-\frac{k}{a}\right),
$$

and it has already been shown that there are systems $T$ and signals $f$ for which the series (2) diverges [10]. For example, the Hilbert transform is a universal system for which there exists a signal such that the peak value of (2) diverges, regardless of the amount of oversampling and the kernel $\phi$. [10].

Here, we analyze the more general, non-equidistant case. In this setting, the sampling-based system approximation process (2) takes the form

$$
\sum_{k=-\infty}^{\infty} f\left(t_{k}\right)\left(T \phi_{k}\right)(t)
$$

where $\left\{t_{k}\right\}_{k \in \mathbb{Z}}$ is the sequence of sampling points and the $\phi_{k}, k \in$ $\mathbb{Z}$, are certain reconstruction functions. An interesting question is whether the additional degree of freedom that is introduced by nonequidistant sampling can be used to circumvent the divergence that was observed in the equidistant setting. In [1] it was conjectured that this is not the case: It was conjectured that the approximation process (3) is not always stable, i.e, that there exist systems $T$ and signals $f$ such that (3) diverges even with oversampling. This conjecture was recently proved in [2].

In this paper consider more general measurement functionals than the pointwise sampling functionals that are used in (3). It will turn out that there exist measurement functionals such that the system approximation process is always stable.

\section{NOTATION}

Let $\hat{f}$ denote the Fourier transform of a function $f$, where $\hat{f}$ is to be understood in the distributional sense. $L^{p}(\mathbb{R}), 1 \leq p<\infty$, is the space of all to the $p$ th power Lebesgue integrable functions on $\mathbb{R}$, with the usual norm $\|\cdot\|_{p}$, and $L^{\infty}(\mathbb{R})$ the space of all functions for which the essential supremum norm $\|\cdot\|_{\infty}$ is finite. $C[a, b]$ denotes the space of all continuous functions on $[a, b]$.

For $\sigma>0$ and $1 \leq p \leq \infty$ we denote by $\mathcal{P W}_{\sigma}^{p}$ the Paley-Wiener space of functions $f$ with a representation $f(z)=$ $1 /(2 \pi) \int_{-\sigma}^{\sigma} g(\omega) \mathrm{e}^{i z \omega} \mathrm{d} \omega, z \in \mathbb{C}$, for some $g \in L^{p}[-\sigma, \sigma]$. If 
$f \in \mathcal{P} \mathcal{W}_{\sigma}^{p}$ then $g(\omega)=\hat{f}(\omega)$. The norm for $\mathcal{P} \mathcal{W}_{\sigma}^{p}, 1 \leq p<\infty$, is given by $\|f\|_{\mathcal{P} \mathcal{W}_{\sigma}^{p}}=\left(1 /(2 \pi) \int_{-\sigma}^{\sigma}|\hat{f}(\omega)|^{p} \mathrm{~d} \omega\right)^{1 / p}$.

We briefly review some definitions and facts about stable linear time-invariant (LTI) systems. A linear system $T: \mathcal{P} \mathcal{W}_{\pi}^{p} \rightarrow \mathcal{P} \mathcal{W}_{\pi}^{p}$, $1 \leq p \leq \infty$, is called stable if the operator $T$ is bounded, i.e., if $\|T\|:=\sup _{\|f\|_{\mathcal{P} \mathcal{W}_{\pi}^{p} \leq 1}}\|T f\|_{\mathcal{P} \mathcal{W}_{\pi}^{p}}<\infty$. Furthermore, it is called time-invariant if $(T f(\cdot-a))(t)=(T f)(t-a)$ for all $f \in \mathcal{P} \mathcal{W}_{\pi}^{p}$ and $t, a \in \mathbb{R}$. For every stable LTI system $T: \mathcal{P} \mathcal{W}_{\pi}^{1} \rightarrow \mathcal{P} \mathcal{W}_{\pi}^{1}$ there exists exactly one function $\hat{h}_{T} \in L^{\infty}[-\pi, \pi]$ such that

$$
(T f)(t)=\frac{1}{2 \pi} \int_{-\pi}^{\pi} \hat{f}(\omega) \hat{h}_{T}(\omega) \mathrm{e}^{i \omega t} \mathrm{~d} \omega, \quad t \in \mathbb{R},
$$

for all $f \in \mathcal{P} \mathcal{W}_{\pi}^{1}$. Conversely, every function $\hat{h}_{T} \in L^{\infty}[-\pi, \pi]$ defines a stable LTI system $T: \mathcal{P} \mathcal{W}_{\pi}^{1} \rightarrow \mathcal{P} \mathcal{W}_{\pi}^{1}$. The operator norm of a stable LTI system $T$ is given by $\|T\|=\|\hat{h}\|_{L^{\infty}[-\pi, \pi]}$. Note that $\hat{h}_{T} \in L^{\infty}[-\pi, \pi] \subset L^{2}[-\pi, \pi]$, and consequently $h_{T} \in \mathcal{P} \mathcal{W}_{\pi}^{2}$.

\section{BASICS OF SYSTEM APPROXIMATION}

In the classical non-equidistant sampling setting the goal is to reconstruct a bandlimited signal $f$ from its non-equidistant samples $\left\{f\left(t_{k}\right)\right\}_{k \in \mathbb{Z}}$, where $\left\{t_{k}\right\}_{k \in \mathbb{Z}}$ is the sequence of sampling points. One possible way to do the reconstruction is to use a sampling series

$$
\sum_{k=-\infty}^{\infty} f\left(t_{k}\right) \phi_{k}(t)
$$

where the $\phi_{k}, k \in \mathbb{Z}$, are certain reconstruction functions.

In this paper we consider sampling point sequences $\left\{t_{k}\right\}_{k \in \mathbb{Z}}$ that are real and a complete interpolating sequence for $\mathcal{P} \mathcal{W}_{\pi}^{2}$.

Definition 1. We say that $\left\{t_{k}\right\}_{k \in \mathbb{Z}}$ is a complete interpolating sequence for $\mathcal{P} \mathcal{W}_{\pi}^{2}$ if the interpolation problem $f\left(t_{k}\right)=c_{k}, k \in \mathbb{Z}$, has exactly one solution $f \in \mathcal{P} \mathcal{W}_{\pi}^{2}$ for every sequence $\left\{c_{k}\right\}_{k \in \mathbb{Z}}$ satisfying $\sum_{k=-\infty}^{\infty}\left|c_{k}\right|^{2}<\infty$.

We further assume that the sequence of sampling points $\left\{t_{k}\right\}_{k \in \mathbb{Z}}$ is ordered strictly increasingly, and, without loss of generality, we assume that $t_{0}=0$. Then, it follows that the product

$$
\phi(z)=z \lim _{N \rightarrow \infty} \prod_{\substack{|k| \leq N \\ k \neq 0}}\left(1-\frac{z}{t_{k}}\right)
$$

converges uniformly on $|z| \leq R$ for all $R<\infty$, and $\phi$ is an entire function of exponential type $\pi$ [11]. It can be seen from (6) that $\phi$, which is often called generating function, has the zeros $\left\{t_{k}\right\}_{k \in \mathbb{Z}}$. Moreover, it follows that

$$
\phi_{k}(t)=\frac{\phi(t)}{\phi^{\prime}\left(t_{k}\right)\left(t-t_{k}\right)}
$$

is the unique function in $\mathcal{P} \mathcal{W}_{\pi}^{2}$ that solves the interpolation problem $\phi_{k}\left(t_{l}\right)=\delta_{k l}$, where $\delta_{k l}=1$ if $k=l$, and $\delta_{k l}=0$ otherwise.

Remark 1. Equidistant sampling with $t_{k}=k, k \in \mathbb{Z}$, is a special case of the more general non-equidistant setting which is considered in this paper. For equidistant sampling we have $\phi_{k}(t)=\operatorname{sinc}(t-k)$, $k \in \mathbb{Z}$, and (5) reduces to the ordinary Shannon sampling series.

Next, we discuss the system approximation process (3) that was introduced in the introduction. For the approximation behavior of the series (3) we have the following negative result, which was proved in [2].
Theorem 1 ([2]). Let $\left\{t_{k}\right\}_{k \in \mathbb{Z}} \subset \mathbb{R}$ be an ordered complete interpolating sequence for $\mathcal{P} \mathcal{W}_{\pi}^{2}, \phi_{k}$ as defined in (7), and $t \in \mathbb{R}$. Then there exists a stable LTI system $T_{*}: \mathcal{P} \mathcal{W}_{\pi}^{1} \rightarrow \mathcal{P} \mathcal{W}_{\pi}^{1}$ such that for every $0<\sigma<\pi$ there exists a signal $f_{*} \in \mathcal{P} \mathcal{W}_{\sigma}^{1}$ such that

$$
\limsup _{N \rightarrow \infty}\left|\sum_{k=-N}^{N} f_{*}\left(t_{k}\right)\left(T_{*} \phi_{k}\right)(t)\right|=\infty
$$

Theorem 1 shows that a digital, i.e., sampling-based, implementation of stable LTI systems is not always possible for the space $\mathcal{P} \mathcal{W}_{\pi}^{1}$. This also illustrates the limits of a general sampling-based technology. However, in the next section we will see that more general measurement functionals can help create a stable approximation process.

\section{SYSTEM APPROXIMATION WITH GENERAL MEASUREMENT FUNCTIONALS}

A key concept in signal processing is to process analog, i.e., continuous-time signals in the digital domain. The fist step in this procedure is to convert the continuous-time signal into a discretetime signal, i.e., into a sequence of numbers. Usually the point evaluation functionals $c_{k}: f \mapsto f\left(t_{k}\right)$ are used to do this conversion. However, it is also possible to use more general measurement functionals $[12,13]$. For example, functionals that take the average of the signal over an interval, like in

$$
c_{k}: f \mapsto \frac{1}{2 \delta} \int_{t_{k}-\delta}^{t_{k}+\delta} f(t) \mathrm{d} t
$$

where $\delta$ is some sufficiently small positive number. In this section we study the system approximation problem for the case that these more general measurement functionals are used instead of the conventional point evaluation functionals.

The approximation of $T f$ by the system approximation process (3) can be seen as an approximation that uses the biorthogonal

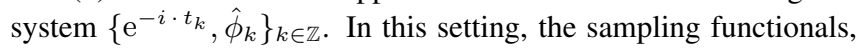
which define a certain measurement procedure, are given by

$$
c_{k}(f)=f\left(t_{k}\right)=\frac{1}{2 \pi} \int_{-\pi}^{\pi} \hat{f}(\omega) \mathrm{e}^{i \omega t_{k}} \mathrm{~d} \omega
$$

and the functions

$$
\phi_{k}(t)=\frac{1}{2 \pi} \int_{-\pi}^{\pi} \hat{\phi}_{k}(\omega) \mathrm{e}^{i \omega t} \mathrm{~d} \omega
$$

serve as reconstruction functions in the approximation process (3).

In Theorem 1 we have seen that for $f \in \mathcal{P} \mathcal{W}_{\pi}^{1}$ even with oversampling an approximation of $T f$ using the process (3) is not possible in general, because there are signals $f \in \mathcal{P} \mathcal{W}_{\pi}^{1}$ and stable LTI systems $T$ such that (3) diverges.

Next, we will study more general measurement procedures than (9) in hopes of circumventing the divergence that was observed in Theorem 1. To this end, we consider a complete orthonormal system $\left\{\hat{\theta}_{n}\right\}_{n \in \mathbb{N}}$ in $L^{2}[-\pi, \pi]$.

For $f \in \mathcal{P} \mathcal{W}_{\pi}^{2}$ the situation is simple. The measurement functionals $c_{n}: \mathcal{P} \mathcal{W}_{\pi}^{2} \rightarrow \mathbb{C}$ are given by

$$
c_{n}(f)=\frac{1}{2 \pi} \int_{-\pi}^{\pi} \hat{f}(\omega) \overline{\hat{\theta}_{n}(\omega)} \mathrm{d} \omega=\int_{-\infty}^{\infty} f(t) \overline{\theta_{n}(t)} \mathrm{d} t .
$$


Further, we have

$$
\lim _{N \rightarrow \infty} \frac{1}{2 \pi} \int_{-\pi}^{\pi}\left|\hat{f}(\omega)-\sum_{n=1}^{N} c_{n}(f) \hat{\theta}_{n}(\omega)\right|^{2} \mathrm{~d} \omega=0
$$

as well as

$$
\lim _{N \rightarrow \infty} \int_{-\infty}^{\infty}\left|f(t)-\sum_{n=1}^{N} c_{n}(f) \theta_{n}(t)\right|^{2} \mathrm{~d} t=0
$$

for all $f \in \mathcal{P} \mathcal{W}_{\pi}^{2}$

In order that

$$
c_{n}(f)=\frac{1}{2 \pi} \int_{-\pi}^{\pi} \hat{f}(\omega) \overline{\hat{\theta}_{n}(\omega)} \mathrm{d} \omega
$$

is also a reasonable measurement procedure for $f \in \mathcal{P} \mathcal{W}_{\pi}^{1}$, we need the functionals $c_{n}: \mathcal{P} \mathcal{W}_{\pi}^{1} \rightarrow \mathbb{C}$, defined by (10), to be continuous and uniformly bounded in $n$. Since

$$
\sup _{\|f\|_{\mathcal{P} \mathcal{W}_{\pi}^{1} \leq 1}}\left|c_{n}(f)\right|=\left\|\hat{\theta}_{n}\right\|_{L^{\infty}[-\pi, \pi]},
$$

this means we additionally have to require that the functions of the complete orthonormal system $\left\{\hat{\theta}_{n}\right\}_{n \in \mathbb{N}}$ satisfy

$$
\sup _{n \in \mathbb{N}}\left\|\hat{\theta}_{n}\right\|_{L^{\infty}[-\pi, \pi]}<\infty .
$$

Using these more general measurement functionals (10), the system approximation process takes the form

$$
\sum_{n=1}^{\infty} c_{n}(f)\left(T \theta_{n}\right)(t) .
$$

The next theorem describes the approximation behavior of (12).

Theorem 2. Let $0<\sigma<\pi$. There exists a complete orthonormal system $\left\{\hat{\theta}_{n}\right\}_{n \in \mathbb{N}}$ in $L^{2}[-\pi, \pi]$ satisfying (11), an associated sequence of measurement functionals $\left\{c_{n}\right\}_{n \in \mathbb{N}}$ as defined by (10), and a constant $C_{1}$ such that for all stable LTI systems $T: \mathcal{P} \mathcal{W}_{\pi}^{1} \rightarrow$ $\mathcal{P W}_{\pi}^{1}$ and all $f \in \mathcal{P} \mathcal{W}_{\sigma}^{1}$ we have

$$
\sup _{t \in \mathbb{R}}\left|\sum_{n=1}^{N} c_{n}(f)\left(T \theta_{n}\right)(t)\right| \leq C_{1}\|f\|_{\mathcal{P} \mathcal{W}_{\sigma}^{1}}\|T\|
$$

for all $N \in \mathbb{N}$, and further

$$
\lim _{N \rightarrow \infty}\left(\sup _{t \in \mathbb{R}}\left|(T f)(t)-\sum_{n=1}^{N} c_{n}(f)\left(T \theta_{n}\right)(t)\right|\right)=0 .
$$

Theorem 2 shows that, using oversampling and more general measurement functionals, it is possible to have a stable system approximation with the process (12). It is interesting to note that Theorem 2 is not only an abstract existence result. The complete orthonormal system $\left\{\hat{\theta}_{n}\right\}_{n \in \mathbb{N}}$ which is used in Theorem 2 can be explicitly constructed by a procedure given in $[14,15]$.

For the proof we need the following theorem from $[14,15]$.

Theorem 3 (Olevskii). Let $0<\delta<1$. There exists an orthonormal system $\left\{\psi_{n}\right\}_{n \in \mathbb{N}}$ of real-valued functions that is closed in $C[0,1]$ such that $\sup _{n \in \mathbb{N}}\left\|\psi_{n}\right\|_{L^{\infty}[0,1]}<\infty$ and such that there exists a constant $C_{2}$ such that for all $x \in[\delta, 1]$ and all $N \in \mathbb{N}$ we have

$$
\int_{0}^{1}\left|\sum_{n=1}^{N} \psi_{n}(x) \psi_{n}(\tau)\right| \mathrm{d} \tau \leq C_{2}
$$

Remark 2. In the above theorem, we adopted the notion of "closed" from [16]. In [16] a system $\left\{\psi_{n}\right\}_{n \in \mathbb{N}}$ is called closed in $C[0,1]$ if every function in $C[0,1]$ can be uniformly approximated by finite linear combinations of the system $\left\{\psi_{n}\right\}_{n \in \mathbb{N}}$, that is if for every $\epsilon>$ 0 and every $f \in C[0,1]$ there exists an $N \in \mathbb{N}$ and a sequence $\left\{\alpha_{n}\right\}_{n=1}^{N} \subset \mathbb{C}$ such that $\left\|f-\sum_{n=1}^{N} \alpha_{n} \psi_{n}\right\|_{L^{\infty}[0,1]}<\epsilon$.

The inequality (13) in Theorem 3 plays a central role in the proof of Theorem 2. If we consider equidistant sampling, as in (1), with the sinc-kernel then, for $a>1$, the series (1) converges globally uniformly. However, a closer look at the expression (13) for the sinc-kernel reveals that

$$
\frac{1}{2 a \pi} \int_{-a \pi}^{a \pi}\left|\sum_{k=-N}^{N} \mathrm{e}^{i k \omega_{1} / a} \mathrm{e}^{-i k \omega / a}\right| \mathrm{d} \omega_{1} \geq \frac{1}{\pi} \log (N)
$$

for all $\omega \in[-\pi, \pi]$. That is, for equidistant sampling and reconstruction with the sinc-kernel, a relation like (13) cannot hold, even with oversampling. This observation is the basis for the divergence result in [10] that was discussed in the introduction.

Proof of Theorem 2. Let $0<\sigma<\pi$ be arbitrary but fixed and set $\delta=(\pi-\sigma) /(2 \pi)$. Using the functions $\psi_{n}$ from Theorem 3, we define

$$
\hat{\theta}_{n}(\omega):=\psi_{n}\left(\frac{\omega+\pi}{2 \pi}\right), \quad \omega \in[-\pi, \pi] .
$$

Due to the properties of the functions $\psi_{n}$, we see that $\left\{\hat{\theta}_{n}\right\}_{n \in \mathbb{N}}$ is a complete orthonormal system for $L^{2}[-\pi, \pi]$, and that

$$
\sup _{n \in \mathbb{N}}\left\|\hat{\theta}_{n}\right\|_{L^{\infty}[-\pi, \pi]}<\infty \text {. }
$$

Furthermore, for $\omega \in[-\sigma, \sigma]$, we have

$$
\begin{aligned}
\frac{1}{2 \pi} \int_{-\pi}^{\pi} & \left|\sum_{n=1}^{N} \hat{\theta}_{n}(\omega) \hat{\theta}_{n}\left(\omega_{1}\right)\right| \mathrm{d} \omega_{1} \\
& =\int_{0}^{1}\left|\sum_{n=1}^{N} \hat{\psi}_{n}\left(\frac{\omega+\pi}{2 \pi}\right) \psi_{n}(\tau)\right| \mathrm{d} \tau \leq C_{2},
\end{aligned}
$$

according to Theorem 3 , because for $\omega \in[-\sigma, \sigma]$ we have $(\omega+$ $\pi) /(2 \pi) \in[\delta, 1]$. Next, we study for $f \in \mathcal{P} \mathcal{W}_{\sigma}^{1}$ the expression

$$
\begin{aligned}
\left(U_{N} \hat{f}\right)(\omega) & :=\sum_{n=1}^{N} c_{n}(f) \hat{\theta}_{n}(\omega) \\
& =\frac{1}{2 \pi} \int_{-\sigma}^{\sigma} \hat{f}\left(\omega_{1}\right) \sum_{n=1}^{N} \hat{\theta}_{n}(\omega) \hat{\theta}_{n}\left(\omega_{1}\right) \mathrm{d} \omega_{1} .
\end{aligned}
$$

We have

$$
\left|\left(U_{N} \hat{f}\right)(\omega)\right| \leq \frac{1}{2 \pi} \int_{-\sigma}^{\sigma}\left|\hat{f}\left(\omega_{1}\right)\right|\left|\sum_{n=1}^{N} \hat{\theta}_{n}(\omega) \hat{\theta}_{n}\left(\omega_{1}\right)\right| \mathrm{d} \omega_{1},
$$

which implies, using Fubini's theorem and (14), that

$$
\begin{aligned}
& \frac{1}{2 \pi} \int_{-\pi}^{\pi}\left|\left(U_{N} \hat{f}\right)(\omega)\right| \mathrm{d} \omega \\
& \leq \frac{1}{2 \pi} \int_{-\sigma}^{\sigma}\left|\hat{f}\left(\omega_{1}\right)\right|\left(\frac{1}{2 \pi} \int_{-\pi}^{\pi}\left|\sum_{n=1}^{N} \hat{\theta}_{n}(\omega) \hat{\theta}_{n}\left(\omega_{1}\right)\right| \mathrm{d} \omega\right) \mathrm{d} \omega_{1} \\
& \leq C_{2}\|f\|_{\mathcal{P} \mathcal{W}_{\sigma}^{1}}
\end{aligned}
$$


Now, let $f \in \mathcal{P} \mathcal{W}_{\sigma}^{1}$ and $\epsilon>0$ be arbitrary but fixed. Then there exists an $f_{\epsilon} \in \mathcal{P} \mathcal{W}_{\sigma}^{2}$ such that

$$
\left\|f-f_{\epsilon}\right\|_{\mathcal{P} \mathcal{W}_{\sigma}^{1}}<\epsilon .
$$

We have

$$
\begin{aligned}
& \frac{1}{2 \pi} \int_{-\pi}^{\pi}\left|\hat{f}(\omega)-\left(U_{N} \hat{f}\right)(\omega)\right| \mathrm{d} \omega \\
& \leq \frac{1}{2 \pi} \int_{-\pi}^{\pi}\left|\hat{f}(\omega)-\hat{f}_{\epsilon}(\omega)\right| \mathrm{d} \omega+\frac{1}{2 \pi} \int_{-\pi}^{\pi}\left|\hat{f}_{\epsilon}(\omega)-\left(U_{N} \hat{f}_{\epsilon}\right)(\omega)\right| \mathrm{d} \omega \\
& \quad+\frac{1}{2 \pi} \int_{-\pi}^{\pi}\left|\left(U_{N}\left(\hat{f}-\hat{f}_{\epsilon}\right)\right)(\omega)\right| \mathrm{d} \omega \\
& \leq \epsilon+C_{2} \epsilon+\left(\frac{1}{2 \pi} \int_{-\pi}^{\pi}\left|\hat{f}_{\epsilon}(\omega)-\left(U_{N} \hat{f}_{\epsilon}\right)(\omega)\right|^{2} \mathrm{~d} \omega\right)^{\frac{1}{2}},
\end{aligned}
$$

where we used (15) and (16). Since $\mathcal{P} \mathcal{W}_{\sigma}^{2} \subset \mathcal{P} \mathcal{W}_{\pi}^{2}$ and $\left\{\hat{\theta}_{n}\right\}_{n \in \mathbb{N}}$ is a complete orthonormal system in $L^{2}[-\pi, \pi]$, there exists a natural number $N_{0}=N_{0}(\epsilon)$ such that

$$
\left(\frac{1}{2 \pi} \int_{-\pi}^{\pi}\left|\hat{f}_{\epsilon}(\omega)-\left(U_{N} \hat{f}_{\epsilon}\right)(\omega)\right|^{2} \mathrm{~d} \omega\right)^{\frac{1}{2}}<\epsilon
$$

for all $N \geq N_{0}$. Hence, we have

$$
\frac{1}{2 \pi} \int_{-\pi}^{\pi}\left|\hat{f}(\omega)-\left(U_{N} \hat{f}\right)(\omega)\right| \mathrm{d} \omega \leq \epsilon\left(2+C_{2}\right)
$$

for all $N \geq N_{0}$. This shows that

$$
\lim _{N \rightarrow \infty} \frac{1}{2 \pi} \int_{-\pi}^{\pi}\left|\hat{f}(\omega)-\left(U_{N} \hat{f}\right)(\omega)\right| \mathrm{d} \omega=0 .
$$

Next, let $T: \mathcal{P} \mathcal{W}_{\pi}^{1} \rightarrow \mathcal{P} \mathcal{W}_{\pi}^{1}$ be an arbitrary but fixed stable LTI system. We have

$$
\begin{aligned}
& (T f)(t)-\sum_{n=1}^{N} c_{n}(f)\left(T \theta_{n}\right)(t) \\
& =\frac{1}{2 \pi} \int_{-\pi}^{\pi}\left(\hat{f}(\omega) \hat{h}_{T}(\omega) \mathrm{e}^{i \omega t}-\sum_{n=1}^{N} c_{n}(f) \hat{h}_{T}(\omega) \hat{\theta}_{n}(\omega) \mathrm{e}^{i \omega t}\right) \mathrm{d} \omega \\
& =\frac{1}{2 \pi} \int_{-\pi}^{\pi}\left(\hat{f}(\omega)-\left(U_{N} \hat{f}\right)(\omega)\right) \hat{h}_{T}(\omega) \mathrm{e}^{i \omega t} \mathrm{~d} \omega
\end{aligned}
$$

and consequently

$$
\begin{aligned}
& \left|(T f)(t)-\sum_{n=1}^{N} c_{n}(f)\left(T \theta_{n}\right)(t)\right| \\
& \quad \leq\left\|\hat{h}_{T}\right\|_{L^{\infty}[-\pi, \pi]} \frac{1}{2 \pi} \int_{-\pi}^{\pi}\left|\hat{f}(\omega)-\left(U_{N} \hat{f}\right)(\omega)\right| \mathrm{d} \omega
\end{aligned}
$$

for all $t \in \mathbb{R}$. From (17) and (18) we see that

$$
\lim _{N \rightarrow \infty}\left(\sup _{t \in \mathbb{R}}\left|(T f)(t)-\sum_{n=1}^{N} c_{n}(f)\left(T \theta_{n}\right)(t)\right|\right)=0 .
$$

Further, we have

$$
\begin{aligned}
\left|\sum_{n=1}^{N} c_{n}(f)\left(T \theta_{n}\right)(t)\right| & \leq \frac{1}{2 \pi} \int_{-\pi}^{\pi}\left|\left(U_{N} \hat{f}\right)(\omega) \hat{h}_{T}(\omega)\right| \mathrm{d} \omega \\
& \leq C_{2}\left\|\hat{h}_{T}\right\|_{L^{\infty}[-\pi, \pi]}\|f\|_{\mathcal{P} \mathcal{W}_{\sigma}^{1}},
\end{aligned}
$$

where we used (15) in the last inequality.
Remark 3. Since $\left\{\hat{\theta}_{n}\right\}_{n \in \mathbb{N}}$ is a complete orthonormal system in $L^{2}[-\pi, \pi]$, it follows that the projections of the functions $\left\{\theta_{n}\right\}_{n \in \mathbb{N}}$ onto $\mathcal{P} \mathcal{W}_{\sigma}^{2}$ form a Parseval frame for $\mathcal{P} \mathcal{W}_{\sigma}^{2}, 0<\sigma<\pi[17$, p. 231]. Thus, Theorem 2 shows that there exist Parseval frames for which we have convergence.

\section{RELATION TO PRIOR WORK}

The approximation of LTI systems by sampling series is a wellstudies field [18, 19, 20, 21, 22, 9], with significance in many areas [23]. Recently, it has been shown that that the system approximation process with classical pointwise sampling can be instable [2]. However, it was already conjectured in [1] that this instability can be overcome by using more general measurement functionals. General measurement functionals have been analyzed before [24, 25, 26, 27], but only for the signal reconstruction problem and not for the system approximation problem. By proving that there exist measurement functionals for which the system approximation process is always stable, we also solved the conjecture from [1].

\section{REFERENCES}

[1] Holger Boche and Ullrich J. Mönich, "Sampling of deterministic signals and systems," IEEE Trans. Signal Process., vol. 59, no. 5, pp. 2101-2111, May 2011.

[2] Holger Boche and Ullrich J. Mönich, "No-go theorem for sampling-based signal processing," in Proceedings of the IEEE International Conference on Acoustics, Speech, and Signal Processing (ICASSP '14), 2014, accepted.

[3] Claude E. Shannon, "Communication in the presence of noise," in Proceedings of the IRE, Jan. 1949, vol. 37, pp. 1021.

[4] Abdul J. Jerri, "The Shannon sampling theorem-its various extensions and applications: A tutorial review," Proc. IEEE, vol. 65, no. 11, pp. 1565-1596, Nov. 1977.

[5] John R. Higgins, "Five short stories about the cardinal series," Bull. Amer. Math. Soc., vol. 12, no. 1, pp. 45-89, 1985.

[6] Farokh Marvasti, Ed., Nonuniform Sampling: Theory and Practice, Kluwer Academic / Plenum Publishers, 2001.

[7] John R. Higgins, Sampling Theory in Fourier and Signal Analysis - Foundations, Oxford University Press, 1996.

[8] P. L. Butzer, W. Splettstößer, and R. L. Stens, “The sampling theorem and linear prediction in signal analysis," Jahresbericht der Deutschen Mathematiker-Vereinigung, vol. 90, no. 1, pp. 1-70, Jan. 1988.

[9] Paul L. Butzer, Gerhard Schmeisser, and Rudolf L. Stens, "Shannon's sampling theorem for bandlimited signals and their Hilbert transform, Boas-type formulae for higher order derivative - the aliasing error involved by their extensions from bandlimited to non-bandlimited signals," Entropy, vol. 14, no. 11, pp. 2192-2226, Nov. 2012.

[10] Holger Boche and Ullrich J. Mönich, "Sampling-type representations of signals and systems," Sampling Theory in Signal and Image Processing, vol. 9, no. 1-3, pp. 119-153, Jan., May, Sept. 2010.

[11] Boris Yakovlevich Levin, Lectures on Entire Functions, AMS, 1996. 
[12] Paul L. Butzer and Junjiang Lei, "Errors in truncated sampling series with measured sampled values for not-necessarily bandlimited functions," Functiones et Approximatio Commentarii Mathematici, vol. 26, pp. 25-39, 1998.

[13] Paul L. Butzer and Junjiang Lei, "Approximation of signals using measured sampled values and error analysis," Communications in Applied Analysis. An International Journal for Theory and Applications, vol. 4, no. 2, pp. 245-255, 2000.

[14] A. M. Olevskii, "Fourier series of continuous functions with respect to bounded orthonormal systems," Izv. Akad. Nauk SSSR Ser. Mat., vol. 30, no. 2, pp. 387-432, 1966.

[15] A. M. Olevskii, "An orthonormal system and its applications," Mat. Sb. (N.S.), vol. 71, no. 113, pp. 297-336, 1966.

[16] A. M. Olevskii, Fourier Series with Respect to General Orthogonal Systems, vol. 86 of Ergebnisse der Mathematik und ihrer Grenzgebiete. 2. Folge, Springer-Verlag, 1975.

[17] Christopher Heil, A Basis Theory Primer: Expanded Edition, vol. 1 of Applied and Numerical Harmonic Analysis, Birkhäuser Boston, 2011.

[18] Henry P. Kramer, "The digital form of operators on bandlimited functions," Journal of Mathematical Analysis and Applications, vol. 44, no. 2, pp. 275-287, Nov. 1973.

[19] Athanasios Papoulis, "Generalized sampling expansion," IEEE Trans. Circuits Syst., vol. 24, no. 11, pp. 652-654, Nov. 1977.
[20] Rudolf L. Stens, "A unified approach to sampling theorems for derivatives and Hilbert transforms," Signal Processing, vol. 5, pp. 139-151, 1983.

[21] Alan V. Oppenheim and Ronald W. Schafer, Discrete-Time Signal Processing, Prentice Hall, 3 edition, 2009.

[22] Muhammad K. Habib, "Digital representations of operators on band-limited random signals," IEEE Trans. Inf. Theory, vol. 47, no. 1, pp. 173-177, Jan. 2001.

[23] Richard P. Feynman, Feynman Lectures on Computation, Penguin Books, 1999.

[24] Wenchang Sun and Xingwei Zhou, "Average sampling in spline subspaces," Applied Mathematics Letter, vol. 15, no. 2, pp. 233-237, Feb. 2002.

[25] Wenchang Sun and Xingwei Zhou, "Reconstruction of bandlimited signals from local averages," IEEE Trans. Inf. Theory, vol. 48, no. 11, pp. 2955-2963, Nov. 2002.

[26] Wenchang Sun and Xingwei Zhou, "Average sampling in shift invariant subspaces with symmetric averaging functions," Journal of Mathematical Analysis and Applications, vol. 287, no. 1, pp. 279-295, Nov. 2003.

[27] Zhanjie Song, Shouyuan Yang, and Xingwei Zhou, "Approximation of signals from local averages," Applied Mathematics Letter, vol. 19, no. 12, pp. 1414-1420, Dec. 2006. 